\title{
Interférences
}

Ars scribendi

$5 \mid 2009$

Historia / Persona

\section{Fonction narrative et idéologique des personnages secondaires dans les Annales de Tacite}

Quelques remarques

\section{Olivier Devillers}

\section{OpenEdition}

Journals

Édition électronique

URL : http://journals.openedition.org/interferences/903

DOI : 10.4000/interferences.903

ISSN : $1777-5485$

\section{Éditeur}

HiSoMA - Histoire et sources des Mondes antiques

Édition imprimée

Date de publication : 1 janvier 2009

ISSN : 1777-5485

\section{Référence électronique}

Olivier Devillers, «Fonction narrative et idéologique des personnages secondaires dans les Annales de Tacite », Interférences [En ligne], 5 | 2009, mis en ligne le 10 juillet 2014, consulté le 15 septembre 2020. URL : http://journals.openedition.org/interferences/903 ; DOI : https://doi.org/10.4000/ interferences. 903

Ce document a été généré automatiquement le 15 septembre 2020

Tous droits réservés 


\title{
Fonction narrative et idéologique des personnages secondaires dans les Annales de Tacite
}

Quelques remarques

\author{
Olivier Devillers
}

1 Dans les Annales, les effets recherchés par l'ensemble des techniques narratives convergent vers les empereurs, qui, seuls, peuvent être tenus comme personnages principaux. Tous les autres sont donc en principe des personnages secondaires ${ }^{1}$, encore que tous ne figurent pas sur le même plan et qu'on ne puisse envisager comme équivalents, par exemple, une épouse impériale et un chevalier simplement nommé dans l'énoncé de témoins à un procès. Afin d'opérer un premier tri, j'ai relevé ceux qui apparaissent de façon active dans au moins trois épisodes différents (un critère évidemment soumis aux aléas de la transmission du texte) ${ }^{2}$. Cette liste, d'environ 95 noms (cf. annexe), est néanmoins sujette à discussion - elle exclut par exemple C. Piso, qui a donné son nom à la conjuration menée contre Néron, mais qui n'apparaît pratiquement que dans cet épisode. Nous en détacherons ici une dizaine de personnages: Germanicus, Thrasea Paetus, Asinius Gallus, Sénèque, Corbulon, Agrippine II, M. Aemilius Lepidus, Cotta Messalinus, Poppaeus Sabinus, M. Ostorius Scapula ${ }^{3}$. Cet échantillon peut être considéré comme représentatif, dans la mesure où il couvre divers modes de relation au prince et diverses époques. En ont été exclus toutefois les non-Romains, dont l'étude se confond avec la question plus générale de l'interaction entre affaires intérieures et extérieures.

2 L'investigation psychologique et le style ont sans doute été les aspects les plus étudiés en relation avec les personnages tacitéens ${ }^{4}$. Nous nous intéresserons plutôt à leur intégration au récit et à leur rôle au service de l'idéologie de l'historien. 


\section{Dimension narrative}

$3 \mathrm{Au}$ portrait ou "portrait-médaillon ", Tacite préfère la caractérisation indirecte, qui révèle une personnalité à travers une série de touches successives qui se complètent et, parfois, se corrigent ${ }^{5}$. L'éparpillement des informations a alors pour conséquence que tel ou tel passage concernant un personnage répond théoriquement à deux types d'impératifs : ceux qui sont liés à la cohérence générale du personnage et ceux qui sont liés au contexte dans lequel est inséré le passage. Si ces deux types d'impératifs entrent en conflit, l'intégration au contexte narratif immédiat l'emporte. C'est là un critère qui distingue le personnage secondaire, dont la représentation offre une certaine plasticité, du personnage principal - Tibère, Néron -, dont la représentation se confond avec la trame narrative. C'est aussi ce qui explique la présence d'incohérences dans le portrait de divers personnages : Germanicus ${ }^{6}$, Agrippine II $^{7}$, Corbulon ${ }^{8} .$.

Dans cette mesure, le recours à la caractérisation indirecte ne peut - du moins pour un personnage secondaire - être tenu pour une garantie d'impartialité, et le personnage, dans sa présentation fragmentée (et forcément fragmentaire), devient un procédé comme un autre de la technique narrative. On le trouvera en association avec d'autres procédés ${ }^{9}$.

5 Le principal est le contraste. Tel est le cas de Germanicus : l'insistance sur sa popularité, notamment, est à comprendre à la lumière de l'impopularité de Tibère. La mise en évidence du même homme lors de ses opérations en Germanie, où il est doté des qualités traditionnelles du bon imperator (celeritas, prudentia...) ${ }^{10}$ et du bon prince (ciuilitas, comitas, modestia...) ${ }^{11}$, va dans le même sens : celui d'un contraste qui traduit sur le plan littéraire une rivalité entre Tibère et Germanicus, ou plus exactement le sentiment de Tibère d'avoir Germanicus pour rival ${ }^{12}$.

6 Corbulon, par son activité aux frontières de l'Empire, fait ressortir les vices de Néron à Rome, ce qui, d'ailleurs, ne repose sur aucune formulation explicite, mais sur le seul art de la composition ${ }^{13}$. Cette fonction est suggérée dès la première apparition du personnage, sous le règne de Claude, lorsque, à propos de ses succès en Germanie, il est caractérisé comme ignauo principi praegrauem (XI, 19, 3: « une lourde charge pour un prince sans courage $»)^{14}$. Corbulon est également opposé à d'autres généraux qui lui semblent inférieurs : surtout Caesennius Paetus dans les livres néroniens, mais aussi Curtius Rufus, au moment de son commandement en Germanie (XI, 21).

7 Thrasea Paetus est également en contraste avec l'empereur, mais sur le plan intérieur. C'est par opposition au prince que sa gloria est fréquemment soulignée (XIII, 49, 3 ; XV, $23,4 ; \mathrm{XVI}, 24,2 ; 25,1 ; 26,3)^{15}$.

8 Un autre procédé est la gradation. Ainsi, A. Malissard voit l'ensemble des deux premiers livres des Annales comme « une sorte d'aristie progressive de Germanicus » ${ }^{16}$. Dans le cas de Thrasea Paetus, cette gradation paraît triple : plus ses qualités sont reconnues, plus il est odieux à Néron et plus le règne de celui-ci se détériore, le procès fait au philosophe constituant un point culminant dans les crimes du prince :

Trucidatis tot insignibus uiris, ad postremum Nero uirtutem ipsam excscindere concupiuit

Après avoir massacré tant d'hommes distingués, Néron finit par concevoir le désir

d'exterminer la vertu elle-même (XVI, 21, 1) ${ }^{17}$.

9 Les apparitions d'Asinius Gallus seront elles aussi regroupées en cinq moments, en écho au développement $\mathrm{du}$ règne de Tibère: débuts contestés, species libertatis, 
développement des procès, répression en deux temps, d'abord non sanglante, puis sanglante. Enfin, la montée progressive des périls se retrouve à travers cinq mentions de Sénèque : il est en butte d'abord sans frais aux critiques de P. Suillius (XIII, 42-43), puis, au prix d'une perte de pouvoir, à des reproches anonymes (XIV, 52, 2-4), puis à l'accusation précise, mais sans suite pour lui, d'un certain Romanus (XIV, 65, 2), puis encore à une tentative d'empoisonnement ordonnée par Néron (XV, 45, 3 ; aussi XV, 60, 2), enfin à l'accusation, qui lui vaudra de devoir se suicider, d'avoir participé à la conjuration de Pison (XV, 56, 2 ; 60-65).

Un troisième procédé est l'archétype, à savoir un modèle de comportement qui s'applique à plusieurs personnages. En I, 74, 2, Tacite énonce celui du délateur : un homme qui, en attaquant les plus illustres citoyens, devient puissant avant de perdre lui-même la vie. Sous Tibère, Fulcinius Trio illustre ce parcours, de sa première apparition comme accusateur de Libo Drusus (II, 28, 2-3) à sa mort, un peu avant celle de Tibère (VI, 38, 2), en passant par son consulat l'année de la chute de Séjan (V, 11).

\section{Dimension idéologique}

11 L'observation des techniques narratives est aussi utile lorsqu'il s'agit d'évaluer les implications idéologiques qui s'attachent à la narration. En effet, au-delà de leur fonction narrative, contrastes, gradations, archétype... supportent une typologie des diverses attitudes face au pouvoir.

\section{Dynastie}

Si Tacite avait voulu présenter Germanicus sous les traits du prince idéal, le moins qu'on puisse dire est qu'il aurait pu être plus efficace ${ }^{18}$ et que la critique moderne ne se partagerait pas entre ceux qui voient dans les Annales un éloge de ce personnage ${ }^{19}$ et ceux qui y voient une critique ${ }^{20}$. Ne peut-on plutôt associer Germanicus à une réflexion sur la dynastie - cette nouvelle réalité qui s'impose à Rome avec le Principat -, son fonctionnement interne (qui en vient à affecter celui de l'Empire), les tensions qu'elle engendre et la manière dont celles-ci sont gérées? Sans doute le bon prince sera-t-il celui qui parvient à maîtriser les tensions inhérentes à l'existence d'héritiers-rivaux au sein de sa propre famille. Tibère n'y réussit pas et cela se répercute sur son image, sur son gouvernement et, au-delà, sur la vie de tous. Tel serait le principal thème de méditation politique auquel est liée la représentation de Germanicus. Ceci est confirmé par le fait qu'après sa mort, son rôle comme source d'instabilité au sein de la dynastie est repris par sa veuve Agrippine $\mathrm{I}^{\mathrm{re}}$ et, dans une certaine mesure, par ses deux fils aînés.

13 L'association avec ce thème de la dynastie est une dimension du portrait d'Agrippine II. L'origine peut s'en trouver dans l'historiographie sénatoriale, ainsi que le suggère l'exploitation de la même idée dans l'Octavie, écrite vraisemblablement au début du règne de Trajan ${ }^{21}$.

Poppée aussi est souvent, surtout si on la compare avec le portrait dressé d'elle par d'autres historiens ${ }^{22}$, considérée sous l'angle de son intrusion dans la vie politique, comme le montre le rôle qui lui est prêté dans les assassinats d'Agrippine II et d'Octavie. 


\section{Cour} dynastie et la cour. Ceci détermine un premier axe de sa présentation: quand il empêche Agrippine de monter sur l'estrade impériale (XIV, 5, 2), au moment de la relation du prince avec Acté (XIII, 13, 1), de la dénonciation d'un prétendu complot d'Agrippine II (XIII, 20, 2), de la prétendue tentative d'inceste avec sa mère (XIV, 2, 1), quand Néron manifeste l'envie de participer à des courses de chevaux (XIV, 14, 2)... Parallèlement, le jugement dont il fait l'objet de la part de Tacite n'a jamais paru bien défini ${ }^{23}$, certains chercheurs évoquant un non liquet ${ }^{24}$. Ces deux aspects seraient liés : il serait impossible, suggérerait Tacite, d'avoir une action politique clairement identifiable en faisant le choix de l'entourage proche du prince. Ceci serait illustré par l'usage qui est fait du thème de la parole en relation avec Sénèque. Tacite se réfère à son eloquentia dès sa première mention pour le règne de Néron en XIII, 2, 1 (praeceptis eloquentiae). Pourtant, d'entrée, la laudatio funebris de Claude par Néron, qu'il a composée, suscite les rires (XIII, 3,1); les fréquents discours qu'il fait prononcer à Néron semblent avoir pour but d'illustrer l'honorabilité de ses préceptes ou de faire briller son esprit (XIII, 11, 1) ; Agrippine II ironise sur sa professoria lingua (XIII, 14, 3) ; la lettre qu'il écrit après la mort de Néron suscite l'hostilité (XIV, 11, 2); ses ennemis lui reprochent de s'arroger à lui seul la gloire de l'éloquence (XIV, 52, 3); le discours par lequel il sollicite sa retraite n'aboutit pas (XIV, 53-56)... En d'autres circonstances encore, Tacite escamote pour ainsi dire sa prise de parole : les accusations que porte contre lui Suillius sont relatées dans un discours indirect (XIII, 42) sans que soit fait écho à une éventuelle réponse; au moment de son trépas encore, comptant sur les ressources de son éloquence, il prononce un discours que Tacite choisit de ne pas reproduire, alléguant qu'il a été publié :

aduocatis scriptoribus, pleraque tradidit, quae, in uulgus edita eius uerbis, inuertere supersedeo

il appela ses secrétaires et prononça un long discours, qui fut publié textuellement et que je m'abstiens de transposer $(\mathrm{XV}, 63,3)$.

Bref, à s'en tenir aux Annales, Sénèque échoue à faire valoir son eloquentia, laquelle est raillée, contestée, inefficace voire inexistante. Or, comme cette eloquentia est par ailleurs incontestable, il faut en conclure que l'incapacité à la faire valoir est imputable aux circonstances mêmes dans lesquelles se place Sénèque, à savoir l'activité de cour. La faillite de son eloquentia vaut comme symbole de sa faillite globale en tant que ministre du prince.

Asinius Gallus ${ }^{25}$, le fils d'Asinius Pollion, ne fait jamais à proprement parler partie de la cour. Il avait cependant épousé Vipsania, la première femme de Tibère, et ce dernier le soupçonnait de nourrir des projets supérieurs à la condition de citoyen (I, 12, 4). Cela même lui sera fatal. Au moment de sa mort, il sera accusé par Tibère d'adultère avec la veuve de Germanicus, Agrippine $\mathrm{I}^{\mathrm{re}}(\mathrm{VI}, 25,2)$, ce qui rejoint le thème de son attraction pour la maison impériale.

Bien entendu, la voie courtisane n'est pas toujours fatale. Cotta Messalinus, auteur comme Asinius Gallus de propositions teintées d'adulation après le procès de Libo Drusus (II, 32, 1-2), critiqué par Tacite (VI, 7, 1), illustre une intimité avec l'empereur qui l'extrait presque des rangs des sénateurs, ainsi qu'il le dit lui-même dans le cadre de son conflit avec M. Lepidus et L. Arruntius ${ }^{26}$ : 
illos quidem Senatus, me autem tuebitur Tiberiolus meus

ils auront, eux, le Sénat pour les défendre, mais moi mon petit Tibère (VI, 5, 1) Sa petite-nièce, Lollia, épousera d'ailleurs Caligula.

Il reste que les cas les plus connus de sénateurs et de nobles qui approchent le pouvoir se soldent par un échec (cf. aussi Séjan). Une formule le résume, qui s'applique au plus emblématique d'entre eux, le seul en tout cas à être devenu prince, Galba :

capax imperii, nisi imperasset

digne de l'Empire, s'il n'avait pas été empereur (H. I, 49, 4)

\section{Sénat}

Si des sénateurs se laissent attirer par la cour, d'autres restent ancrés au Sénat. L'époque de Tibère se prête particulièrement à évoquer ces derniers. Tacite utilise alors le fonds documentaire fourni par les acta Senatus et nombre de sénateurs sont des figures récurrentes des Annales I-VI : parmi eux, M. Aemilius Lepidus, mentionné à plusieurs reprises et dont Tacite lui-même considère que la caractérisation indirecte dans les livres tibériens met en avant la sagesse :

obiit eodem anno et M. Lepidus, de cuius moderatione atque sapientia in prioribus libris satis conlocaui

la même année mourut aussi $\mathrm{M}$. Lepidus, dont j'ai assez montré la modération et la sagesse dans les livres précédents (VI, 27, 4) ${ }^{27}$.

Dès sa première mention, Tacite signale qu'il réussit à échapper à toute accusation sous Tibère et c'est à son propos qu'il formule son éloge du chemin entre résistance et adulation (IV, 20,3). Or cet éloge naît précisément en opposition avec l'attitude de deux hommes cités ci-dessus, Asinius Gallus, qui vient de proposer de confisquer une partie des biens d'un accusé (IV, 20, 1-2), et Cotta Messalinus, qui suggère également une mesure rigoureuse $(\mathrm{IV}, 20,4)^{28}$. De même, lorsqu'il passe en revue les capaces imperii au crépuscule du règne d'Auguste, Tacite fait figurer à la suite $M$. Lepidus qui est compétent pour exercer le pouvoir, mais ne le souhaite pas, et Asinius Gallus qui le souhaite sans en avoir la compétence (I, 13,2). Reviendrait alors l'idée d'opposer un sénateur qui, attiré par la cour, va à sa perte (Asinius) et un autre (Lepidus) qui s'honore en restant fidèle aux prérogatives traditionnelles de sa classe, ainsi lorsqu'il accepte d'assister un accusé (III, 11, 2), lorsqu'il restaure à ses frais la basilique de Paulus, monument de sa famille (III, 72, 1), ou dans son activité de gouverneur de province (IV, 56, 3)... ; l'insistance sur son illustration familiale est en outre un trait de sa notice nécrologique (VI, 27) ${ }^{29}$. S'il y a une ombre dans sa représentation, elle concerne précisément un rapprochement avec la dynastie, à savoir le mariage de sa fille avec Drusus III, contre lequel elle lança des accusations qui lui valurent d'être abhorrée, même si, aussi longtemps que vécut son père, elle resta impunie (VI, 40,3).

Pour l'époque néronienne, Lepidus trouve un équivalent en Thrasea Paetus ${ }^{30}$. L'intervention de ce dernier lors du procès d'Antistius Sosianus (XIV, 48-49) est ainsi à rapprocher de celle de Lepidus au procès de Clutorius Priscus (III, 50); dans les deux cas, il s'agit pour le Sénat de s'exprimer sur le sort d'un homme contre lequel a été requise la peine de mort pour avoir porté atteinte à la majesté du prince. Parallèlement, un jeu d'analogies et de rappels avec Sénèque précise le rôle de Thrasea au sein du règne néronien: alors que l'action de Sénèque est systématiquement envisagée dans son rapport avec la domus Caesaris, le champ d'activité de Thrasea s'identifie avec le Sénat. Il peut ainsi servir de vecteur à la réflexion sur la libertas. Le 
trait, et même le mot, revient dans la plupart de ses interventions (XIII, 49, 2 ; XIV, 12, $11 ; 49,1 ; \mathrm{XVI}, 22,4 ; 24,2)^{31}$. Pourtant, si on le compare à Lepidus, Thrasea a eu une mort violente, ce qui, narrativement, traduirait une évolution entre le règne de Tibère, où l'homme vertueux peut esquiver les périls, et celui de Néron, durant lequel cela est devenu impossible ${ }^{32}$.

Enfin, au sein même des livres néroniens, Thrasea a son pendant, pour les affaires militaires et extérieures, en Corbulon ${ }^{33}$. Dès sa première opération en Germanie, celuici fait montre d'une asperitas etiam aduersus leuia (XI, 18, 3: " une rigueur même à l'égard des fautes légères ») qui fait songer à l'attitude dont se réclame Thrasea au début du règne de Néron, lorsqu'il intervient à propos du nombre de gladiateurs permis aux Syracusains lors de leurs jeux :

ut manifestum fieret magnarum rerum curam non dissimulaturos qui animum etiam leuissimis aduerterent

afin de montrer clairement que ne fermeraient pas les yeux sur les affaires

imortantes ceux qui prêtaient attention même aux plus futiles (XIII, 49, 4) ${ }^{34}$

une expression qui trouve elle-même un écho dans la position prise par Néron à ses débuts :

ut iuuenilis animus, leuium quoque gloria sublatus, maiores continuaret

afin de pousser cette âme juvénile, exaltée par la gloire qui s'attachait même aux petites choses, à en réaliser de plus grandes par la suite (XIII, 11, 1).

Comme Thrasea, également, Corbulon se tient à l'écart des cercles proches du pouvoir ; aucun des passages que nous avons conservés ne le montre en train d'intervenir sur la scène intérieure. Dès lors, le contraste entre Corbulon et Néron, même s'il peut suggérer une rivalité potentielle, n'est pas à considérer sur le même plan que la relation entre Germanicus et Tibère; dégagé de la dimension dynastique, il exprime plutôt les difficultés du rapport entre le prince et ses généraux, membres des classes supérieures.

S'il fallait trouver, pour le règne de Tibère, un équivalent à Corbulon, il faudrait considérer un sénateur uniquement signalé dans son rapport avec les provinces. Tel est Poppaeus Sabinus, qui fut longtemps gouverneur de province et dont sont assez longuement narrées les opérations en Thrace (IV, 46-51). La notice nécrologique qui lui est consacrée (VI, 39, 3) met en évidence ce fait, mais indique également - ce qui le différencie de Corbulon, sur le même mode que Lepidus se différencie de Thrasea - qu'il ne fut pas en butte à l'hostilité des princes : signe que de telles carrières étaient encore possibles sous Tibère (alors qu'elles cesseront de l'être sous Néron), mais aussi conséquence du fait que Poppaeus ne semble pas avoir autant de mérites que Corbulon.

Bien sûr, il est possible qu'un même sénateur apparaisse à la fois sur le plan extérieur et sur le plan intérieur. Il en va ainsi de M. Ostorius Scapula: le fait qu'il obtient sous Claude la couronne civique lors des campagnes en Bretagne (XII, 31, 4) le rapproche de Corbulon, tandis que la position qu'il occupe lors du procès d'Antistius Sosianus (XIV, 48, 1-2) le place du côté de Thrasea. Cumulant ainsi les traits de deux hommes qui finirent par s'attirer la colère du prince, c'est sans surprise qu'il est lui aussi contraint à se donner la mort (XVI, 14-15); la réputation militaire dont il jouissait, et que Néron redoutait, est rappelée dans ce contexte (XVI, 15, 1).

En somme, le portrait des personnages est entièrement intégré à l'arsenal des techniques narratives de Tacite; on pourrait presque dire qu'il est une technique narrative lui-même. Dans cette mesure, il participe à la portée idéologique de l'œuvre, à travers la mise en place de «types » qui répondent à autant de façons de se situer par 
rapport au prince. Deux pôles se dégagent alors: d'un côté, la dynastie, dont les membres (Germanicus, Agrippine II) n'échappent pas aux tensions souvent délétères du pouvoir, de l'autre, les membres de la noblesse qui se cantonnent dans la tradition et les prérogatives de leur classe, sans doute plus exposés sous Néron (Thrasea Paetus, Corbulon, M. Ostorius Scapula) que sous Tibère (Aemilius Lepidus, Poppaeus Sabinus). Entre ces deux pôles, des hommes qui cèdent à l'attraction de la cour et tendent à une plus grande familiarité, soit par des conseils politiques, soit par des alliances matrimoniales, avec le pouvoir: voie difficile qui, si elle profite à certains (Cotta Messallinus), s'avère fatale pour d'autres, tant sous Tibère (Asinius Gallus) que sous Néron (Sénèque).

\section{BIBLIOGRAPHIE}

ALEXANDER W.H. 1952, The Tacitean 'Non Liquet' on Seneca, University of California Publications in Classical Philology 14, 8, Berkeley - Los Angeles.

AUBRION É. 1985, Rhétorique et histoire chez Tacite, Metz.

AUBRION É. 1991, «L'eloquentia de Tacite et sa fides d'historien », ANRW, II, 33, 4, p. 2597-2688.

BOISSIER G. 1904, Tacite, $2^{\mathrm{e}}$ éd., Paris.

BORZSÁK S. 1969, « Das Germanicusbild des Tacitus », Latomus 28, p. 588-600.

BORZSÁK S. 1970, « Zum Verständnis der Darstellungskunst des Tacitus. Die Veränderungen des Germanicus-Bildes », AAntHung 18, p. 279-292.

BORzsÁK S. 1982, « Alexander der Gr. als Muster taciteischer Heldendarstellung », Gymnasium 89, p. 37-56.

BOsWORTH A.B. 1977, « Tacitus and Asinius Gallus », AJAH 2, p. 173-192.

DAITZ S.G. 1960, « Tacitus' Technique of Character Portrayal », AJPh 81, p. 30-52.

DEVILLERS O. 1994, L'art de la persuasion dans les Annales de Tacite, Coll. Latomus 223, Bruxelles.

DEVILLERS 0. 2002, «Le rôle des passages relatifs à Thrasea Paetus dans les Annales de Tacite », in J.-M. Croisille, Y. Perrin (éds), Neronia VI : Rome à l'époque néronienne. Institutions et vie politique, économie et société, vie intellectuelle, artistique et spirituelle, Coll. Latomus 268, Bruxelles, p. 296-311. DEVILLERS 0. 2005, « Le thème de la famille bouleversée dans l'Octavie », VL 172, p. 33-40.

GEISER M. 2007, Personendarstellung bei Tacitus am Beispiel von Cn. Domitius Corbulo und Ser. Sulpicius Galba, Die Antike und ihr Weiterleben 6, Remscheid.

HELDMANN K. 1991, « Libertas Thraseae servitium aliorum rupit. Überlegungen zur Geschichtsauffassung im Spätwerk des Tacitus », Gymnasium 98, p. 207-231.

HENRY D., WALKER B. 1963, « Tacitus and Seneca », G\&R 10, p. 98-110.

HOLZTRATTNER F. 1995, Poppaea Neronis potens: die Gestalt der Poppaea Sabina in den Nerobüchern des Tacitus. Mit einem Anhang zu Claudia Acte, Grazer Beiträge. Supplementband 6, Graz - Horn. 
KROHN F. 1934, Personendarstellung bei Tacitus [Diss. Leipzig].

LEHMANN G.A. 1971, « Tacitus und die imitatio Alexandri des Germanicus Caesar. Politik und literarische Kunst im Werk des Tacitus », AU 14, p. 23-36.

MALISSARD A. 1990, «Germanicus, Alexandre et le début des Annales de Tacite. À propos de Tacite, Annales, 2, 73 », in J.-M. Croisille (éd.), Neronia IV : Alejandro Magno, modelo de los emperadores romanos, Coll. Latomus 209, Bruxelles, p. 328-338.

MCCULLOCH H.Y. 1984, Narrative Cause in the Annals of Tacitus, Beiträge zur klassischen Philologie 160, Königstein/Ts.

MELLOR R. 1993, Tacitus, New York - Londres.

MENDELL C.W. 1957, Tacitus: The Man and His Work, New Haven - Londres.

MICHEL A. 1966, Tacite et le destin de l'Empire, Coll. Signes des temps 18, Paris.

PALADINI M.L. 1984, «A proposito del parallelo Alessandro Magno-Germanico Cesare in Tacito », in M. Sordi (éd.), Alessandro Magno tra storia e mito, Ricerche dell'Istituto di storia antica dell'Università cattolica di Milano 1. Edizioni universitarie Jaca 2, Milan, p. 179-193.

PARATORE E. 1952, « La figura di Agrippina minore in Tacito », Maia 5, p. 32-81.

PIGON J. 2008, «The Passive Voice of the Hero: Some Pecularities of Tacitus'Portrayal of Germanicus in Annals 1.31-49 ", in Id., The Children of Herodotus: Greek and Roman Historiography and Related Genres, New Castle, p. 287-303.

ROSs D.O. 1973, « The Tacitean Germanicus », YClS 33, p. 209-227.

RUBIÈS J.P. 1994, « Nero in Tacitus and Nero in Tacitism: the Historian's Craft », in J. Masters, J. Elsner (éds), Reflections of Nero: Culture, History \& Representation, Londres, p. 29-47.

SAILOR D. 2008, Writing and Empire in Tacitus, Cambridge - New York.

SHOTTER D.C.A. 1968, « Tacitus, Tiberius und Germanicus », Historia 17, p. 194-214.

SHOTTER D.C.A. 1988, « Tacitus and Tiberius », AncSoc 19, p. 225-236.

SINCLAIR P. 1995, Tacitus the Sententious Historian: A Sociology of Rhetoric in Annales 1-6, University Park (Pa.).

SYME R. 1958, Tacitus, Oxford Books, Oxford.

\section{ANNEXES}

Personnages mentionnés de façon significative dans trois épisodes au moins des Annales

\section{Règne de Tibère}

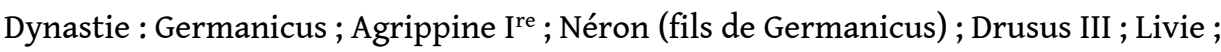
Drusus II ; Livia (épouse de Drusus II)

Cour : Séjan; Macron ; Plancine

Sénat : Cn. Piso ; L. Piso ; M. Aemilius Lepidus ; Mam. Aemilius Scaurus ; Asinius Gallus ; L. Arruntius ; Iullus Antonius ; A. Caecina Severus ; P. Cornelius Dolabella ; Cotta Messalinus ; Fulcinius Trio ; Q. Haterius ; D. Haterius Agrippa ; Iunius Blaesus père ; Iunius Blaesus fils ; M. Iunius Silanus ; L. Pomponius Flaccus ; Poppaeus Sabinus ; 
C. Rubellius Blandus ; Satrius Secundus ; Seius Strabo ; Q. Servaeus ; C. Silius père ; L. Stertinius ; P. Sulpicius Quirinus ; Titius Sabinus ; Trebellenus Rufus ; C. Vibius Serenus; P. Vitellius

Règnes de Tibère et de Claude

Sénat : L. Apronius; Vibius Marsus (surtout prééminents pour le règne de Tibère) ; App. Iunius Silanus ; P. Pomponius Secundus ; L. Vitellius

Règnes de Tibère et de Néron

Sénat : Domitius Afer

Règnes de Tibère, Claude et Néron

Dynastie : Agrippine II

Sénat: P. Memmius Regulus; P. Suillius Rufus; Q. Veranius

Règne de Claude

Dynastie : Messaline

Cour : Narcisse

Sénat : C. Silius fils

\section{Règnes de Claude et de Néron}

Dynastie : Britannicus ; Octavie ; Antonia (fille de Claude) ; Iunia Silana

Cour : Pallas ; Burrus ; Sénèque

Sénat : Corbulon ; C. Cassius ; Cossutianus Capito ; Faustus Cornelius Sulla ; Eprius

Marcellus ; Faenius Rufus ; Helvidius Priscus ; D. Iunius Silanus Torquatus ; M. Ostorius Scapula ; Plautius Lateranus ; Rufrius Crispinus ; Ummidius Quadratus ; L. Volusius fils

Règne de Néron

Dynastie : Poppée

Cour : Tigellin; Othon

Sénat : Thrasea Paetus ; Caesennius Paetus ; Antistius Sosianus ; L. Antistius Vetus ;

Rubellius Plautus

\section{Non-Romains}

Arminius ; Artaban ; Artaxias ; Cotys ; Inguiomer ; Maroboduus ; Mithridatès (Hiberus) ; Pharasmanès ; Phraatès ; Radamiste ; Rhoemetalcès ; Tacfarinas ; Tigrane V ; Tiridatès ;

Vologèse ; Vononès

\section{NOTES}

1. Dans Mendell 1957, p. 143, la distinction entre "major» et "minor characters" ne s'accompagne pas d'une réflexion sur les critères permettant de les distinguer.

2. C'est clairement le cas de Corbulon, dont la mort était relatée dans la fin (perdue) des Annales et qui, aussi, avait sans doute été mentionné avant son apparition (la première pour nous) en XI, 18, 1 (Geiser 2007, p.31-32). Une mention antérieure aux livres néroniens n'est pas non plus exclue dans le cas de Thrasea Paetus (Aubrion 1985, p. 430).

3. D’autres seront mentionnés plus indirectement: la famille de Germanicus, Poppée, Fulcinius Trio...

4. Par Krohn 1934 ; Daitz 1960.

5. P. ex. Aubrion 1985, p. 28, 385-386, 480 et 486 ; Aubrion 1991, p. 2640-2642. Ce type de caractérisation indirecte domine aussi dans les Histoires; Syme 1958, p. 196. 
6. Aubrion 1985, p. 428-430; Aubrion 1991, p. 2635. Aussi Malissard 1990, p. 333, qui parle de "réseau assez dense, et bien connu, de contradictions".

7. Ainsi entre XII, 7, 3 : nihil domi impudicum et 64,5 (à propos aussi de Domitia Lepida) : utraque impudica ; déjà Boissier 1904, p. 28-29. Pour sa part, Paratore 1952, p. 81, voit ces contradictions comme le reflet de la personnalité, contradictoire, d'Agrippine II elle-même.

8. Geiser 2007, p. 14-15.

9. Sur ceux-ci, Devillers 1994, p. 97-184.

10. Pour la celeritas, I, 50, 1 et $3 ; 56,2-3$; II, 8, 4 ; 25, 2. Pour la prudentia, I, 51, 2 ; II, 20, 1. Aussi le rapprochement avec Alexandre: I, 61, 1 ; II, 21, 2 ; 24, 2 ; 53 ; 61, 2 ; 73. Par ex. Borzsák 1969 ; Borzsák 1970 ; Borzsák 1982 ; Lehmann 1971 ; Paladini 1984 ; Malissard 1990.

11. Pour la ciuilitas et la comitas, I, 33, 2 ; 71, 3 ; II, 13, $1 ; 55,1 ; 72,2 ; 82$, 2. Pour la modestia, II, 22, $1 ; 26,4 ; 58,2$.

12. Cf. I, 7,$6 ; 52,1$. Ce sentiment prend parfois la forme d'une hostilité de l'empereur envers Germanicus : II, 5, 1 ; 26, 5 ; IV, 1,1 .

13. Geiser 2007, p. 145-147.

14. Les traductions sont celles de la CUF, parfois quelque peu modifiées.

15. Devillers 2002, p. 308.

16. Malissard 1990, p. 334.

17. Devillers 2002, p. 300-303.

18. McCulloch 1984, p. 11. Parmi les passages qui ont été interprétés comme peu flatteurs envers Germanicus, I, 4, 5; 34-35; 36, 3 ; 39, 3 ; 40, 1 et 3 ; 44-49 (spécialement ses larmes en 49, 2) ; 78, 2 ; II, 8,$2 ; 51$.

19. Par ex. Michel 1966, p. 121-130; Aubrion 1985, p. 188, 422 et 429-430.

20. Shotter 1968 ; Shotter 1988, p. 232-234; Ross 1973. Sur cette division de la critique, encore par ex. Pigon 2008, p. 289-290.

21. Devillers 2005.

22. Holztrattner 1995.

23. Henry, Walker 1963, p. 107 : «Seneca in the Annals remains so colourless and indeterminate a character $"$.

24. Alexander 1952.

25. Bosworth 1977.

26. Il est encore opposé à L. Arruntius en VI, 7, 1.

27. Sur le portrait tacitéen de Lepidus, Sinclair 1995, p. 164-184.

28. L'opposition avec Cotta Messalinus est également explicite en VI, $5,1$.

29. Aussi III, 72, 1 ; IV, 20, 4.

30. À propos de ce dernier, également, les chercheurs n'ont pu s'accorder sur le jugement porté par Tacite : favorable ou défavorable, la critique de l'historien portant alors non sur le courage de l'homme, mais sur ses options politiques. Dans le sens d'un jugement plutôt défavorable, Aubrion 1985, p. 431-432, 675 ; pour une critique essentiellement politique, par ex. Mellor 1993, p. 50, 75 ; aussi Sailor 2008, p. 19-23 ; dans le sens d'un jugement plutôt favorable, y compris politiquement, Heldmann 1991, p. 207-231.

31. Aussi XVI, 35, 1, offrande à Jupiter Libérateur.

32. Devillers 2002, p. 309-310 ; aliter Sailor 2008, p. 28, n. 62.

33. Dans ce sens, Rubiès 1994, p. 40. Sur les différentes opinions relatives au jugement de Tacite sur Corbulon, bibliographie par Geiser 2007, p. 16, n. 25-26.

34. Déjà XIII, 9, 2 : tam leuia consectaretur. 


\section{RÉSUMÉS}

L'article examine quelques personnages secondaires dans les Annales de Tacite en s'intéressant à leur intégration au récit et à leur rôle au service de l'idéologie de l'historien. Il examine les personnages de Germanicus, Thrasea Paetus, Asinius Gallus, Sénèque, Corbulon, Agrippine II, M. Aemilius Lepidus, Cotta Messalinus, Poppaeus Sabinus, M. Ostorius Scapula. Le portrait de ces personnages est entièrement intégré à l'arsenal des techniques narratives de Tacite ; on pourrait presque dire qu'il est une technique narrative lui-même. Dans cette mesure, il participe à la portée idéologique de l'œuvre, à travers la mise en place de «types » qui répondent à autant de façons de se situer par rapport au prince. Deux pôles se dégagent alors : d'un côté, la dynastie, dont les membres (Germanicus, Agrippine II) n'échappent pas aux tensions souvent délétères du pouvoir, de l'autre, les membres de la noblesse qui se cantonnent dans la tradition et les prérogatives de leur classe, sans doute plus exposés sous Néron (Thrasea Paetus, Corbulon, M. Ostorius Scapula) que sous Tibère (Aemilius Lepidus, Poppaeus Sabinus). Entre ces deux pôles, des hommes qui cèdent à l'attraction de la cour et tendent à une plus grande familiarité, soit par des conseils politiques, soit par des alliances matrimoniales, avec le pouvoir : voie difficile qui, si elle profite à certains (Cotta Messallinus), s'avère fatale pour d'autres, tant sous Tibère (Asinius Gallus) que sous Néron (Sénèque).

\section{INDEX}

Mots-clés : histoire et idéologie, historiographie romaine, historiographie sénatoriale, idéologie dynastique, idéologie impériale romaine, morale et littérature, narration, personnages types, prosopographie impériale romaine, Sénat romain

nomsmotscles Octavie, Tacite

Keywords : history and ideology, Roman historiography, senatorial historiography, dynastic ideology, Roman imperial ideology, ethics and literature, narrative, stock characters, Roman imperial prosopography, Roman senate

\section{AUTEURS}

\section{OLIVIER DEVILLERS}

Université Bordeaux III - UMR 5607 